\title{
Re: Nocturia Improvement with Surgical Correction of Sleep Apnea
}

\author{
Park HK1, Paick SH1, Kim HG1', Park DH², Cho JH33, Hong SC33, Choi WS1 \\ ${ }^{1}$ Konkuk University Faculty of Medicine, Department of Urology, Seoul, Korea \\ ${ }^{2}$ Konkuk University Faculty of Medicine, Department of Neuropsychiatry, Seoul, Korea \\ ${ }^{3}$ Konkuk University Faculty of Medicine, Department of Otorhinolaryngology-Head and Neck Surgery, Seoul, Korea \\ Int Neurourol J 2016;20:329-334. doi: 10.5213/inj.1632624.312.
}

\section{EDITORIAL COMMENT}

As a common lower urinary tract symptom (LUTS), nocturia also reduces the quality of life (QoL) and is associated with sleep disruption and daytime fatigue. In an epidemiological study, the prevalence of nocturia was $49 \%$ in men and $55 \%$ in women. Nocturia has usually been managed as an isolated symptom, with therapy based on LUTS, including alpha blockers and anticholinergic drugs. Recent research suggests, however, that the etiology of nocturia differs from that of other LUTSs, and that nocturia should be evaluated as a separate condition, as well as a component of a systemic disease, such as obstructive sleep apnea (OSA) syndrome. Increased atrial natriuretic peptide secretion, increased urinary production, and increased alpha sympathetic nervous activity in patients with OSA can also be responsible for LUTS, especially nocturia. In this study, the authors speculated that correction of OSA would be expected to reduce the severity of nocturia. Of 256 patients diagnosed with OSA, 66 patients planned to undergo uvulopalatopharyngoplasty surgery for correction of OSA were included in this study. The number of nocturia episodes was evaluated by the International Prostate Symptom Score (IPSS). As a result, overall voiding and storage parameters including nocturia were improved after OSA correction surgery and the nocturia episodes were significantly decreased from $1.8 \pm 1.1$ to $0.8 \pm 1.2(p<0.001)$. Total mean IPSS was decreased from $9.3 \pm 6.9$ to $5.9 \pm 5.9(p<0.001)$. The authors concluded that OSA correction surgery improved LUTS, QoL, and nocturia episodes. Patients with nocturia should be questioned about signs and symptoms of snoring and apnea and referred for surgical correction if indicated.

Metin Onaran, MD

๑Copyright 2017 by the Association of Urological Surgery / Journal of Urological Surgery published by Galenos Publishing House. 